\title{
Effects of Quince Seed Mucilage and Green Tea Extract as Active Edible Coatings on Quality of Pacific White Shrimps During Cold Storage
}

\author{
Mohammad Noshad ${ }^{* 1}$, Behzad Nasehi ${ }^{2}$, Adieh Anvar ${ }^{1}$ \\ 1- Department of Food Science and Technology, Faculty of Animal Science and Food Technology, Agricultural Sciences and Natural Resources \\ University of Khuzestan, Mollasani, Iran \\ 2- Department of Agricultural Engineering and Technology, Payame Noor University (PNU), Iran
}

\section{A B S T R A C T}

Background and Objectives: Shrimps (Litopenaeus vannamei) include high market prices and nutritional values; therefore, they are important aquatic resources worldwide. However, the shelf life of shrimps is limited due to microbiological deterioration during their storage. In recent years, several methods have been used to improve the quality of shrimps during cold storage. One of the most important methods includes use of edible coatings. Therefore, the objective of this study was to assess effects of active edible coatings from quince seed mucilage and green tea extract (as an antimicrobial antioxidant compounds) on physicochemical and quality properties of shrimps during cold storage.

Materials and Methods: This study assessed effects of quince seed mucilage (QSM) coating combined with green tea extract (GTE) on color, $\mathrm{pH}$, total volatile basic nitrogen (TVB-N), texture and microbial quality of the Pacific white shrimps (Litopenaeus vannamei) within 10 days of cold storage.

Results: Results showed that $\mathrm{pH}$ and TVB-N values significantly increased $(p<0.05)$ in shrimps treated with QSM coating combined with GTE. Hardness and springiness of the shrimps were improved by increasing the concentration of GTE, compared to controls. Total aerobic plate counts of the shrimps treated by QSM coating combined with GTE were lower than those of controls. The total color difference $(\Delta \mathrm{E})$ of the controls was lowest than that of other treatments at the end of the storage time. The $a^{*}$ value of the shrimps decreased from $-0.87 \pm 0.21$ to $-5.05 \pm 1.08$ (showing the attitude to greenness) and the $\mathrm{b}^{*}$ values increased from $1.44 \pm 0.84$ to $16.47 \pm 1.61$ (showing the attitude to yellowness).

Conclusions: These results have suggested that the QSM and GTE coating can decrease spoilage and decomposition that cause quality loss of shrimps.

Keywords: Quince, Green tea, Shrimp, Bioactive coating, Microbial activity

\section{Introduction}

Shrimps (Litopenaeus vannamei) are expensive in markets and include high nutritional values, therefore, they are included in the most important aquatic nutritional resources in world $(1,2)$. However, storage time of shrimps is limited due to microbiological deterioration. Thus, shrimps are mostly difficult to store within the seafoods (3). Currently, several shrimp preservation technologies are used, including low-temperature preservation, modified atmosphere packaging, chemical preservation and biological preservation. Of these preservation methods, the most widely used method is the ow-temperature preservation. In low-temperature storage, fresh shrimps are stored at $0-4{ }^{\circ} \mathrm{C}$. Unpleasant odor development in shrimps is one of the most important problems that occur when shrimps are stored at low temperatures. This occurs because temperature is not low enough; hence, high temperatures cause spoilage of the shrimps more quickly, resulting in unpleasant odors (4-6). In recent years, several methods have been used to improve the quality of shrimps during cold storage. Use of edible coating is one of the most important technologies that are suggested (7-10). In this study, a novel hydrocolloid was investigated for this mean, including quinc e seed mucilage (QSM) and a mixed 
solution of QSM and green tea extract (GTE) as novel edible coatings. A complex of a cellulosic fraction with a further readily hydrolyzed polysaccharide such as arabinose and xylose were contents of the QSM $(11,12)$. These polysaccharides included unique colloidal properties, low production costs and easy extraction rates are appropriate for edible coatings and films (13). Green tea (Camellia sinensis) is an excellent source of polyphenolic compounds such as catechins, theaflavins and thearubigins with strong antioxidant and antimicrobial properties $(14,15)$. Therefore, the purpose of this study was to assess effects of active edible coatings from QSM and GTE (as an antimicrobial antioxidant compound) on physicochemical and quality properties of the shrimps during cold storage.

\section{Materials and Methods}

Fresh, non-treated Pacific white shrimps ( $L$. vannamei) were purchased from the local shrimp market in Ahvaz, Iran. The average weight of shrimps was $20-22 \mathrm{~g}$. The quince seeds and green teas were purchased from a local grocery in Ahvaz, Iran. All chemicals were purchased from Merck (Darmstadt, Germany) with analytical grades.

\section{Preparation of the coating solutions}

Quince seeds were crushed based on a method by Jouki et al. (2014). After sieving the quince seeds $(\sim 10 \mathrm{~g})$, they were washed with three folds of ethanol $(96 \% \mathrm{w} / \mathrm{v})$ for $5 \mathrm{~min}$ with constant agitating. After ethanol separation, seeds were dried at $45{ }^{\circ} \mathrm{C}$. Distilled water (D.W., water to seed ratio of 30:1) was used to separate aqueous mucilage from the whole seeds. This was then filtered using cheese cloth (13). Method of Sabaghi et al. (2015) was used to extract the green teas. Briefly, blender (Moulinex 320, Spain) was used to ground the green tea leaves. This was then sieved through a mesh ( 0.23 -mm sieve size). The GTE solutions were prepared By blending green tea powder with D.W. (1:5 w/w) and heating for $15 \mathrm{~min}$ at $80{ }^{\circ} \mathrm{C}$ using water bath (Heraeus, Germany) to maximize the extraction of phenolic compounds. Whatman No. $1(11 \mathrm{~mm})$ filter papers were used to filter the GTE solutions. Various concentrations of GTE (0-20\%) were mixed to achieve five coating solutions of QG 0-4 (15). An uncoated sample was used as control.

\section{Shrimp coating}

Shrimps were uniformly soaked in coating solutions for $40 \mathrm{~s}$. Furthermore, shrimps were dipped in D.W. as control samples. Samples were dried at $25^{\circ} \mathrm{C}$ after coating process. All treatments were packaged individually in sterile polyethylene bags and stored at $4{ }^{\circ} \mathrm{C}$ for $10 \mathrm{~d}$.

Table 1. Formulations of the shrimp treatments GTE, green tea extract

\begin{tabular}{l}
\hline Treatment \\
\hline Uncoated (Control) \\
Coating with QSM with no GTE (QG-0) \\
Coating with QSM and 5\% of GTE (QG-1) \\
Coating with QSM and 10\% of GTE (QG-2) \\
Coating with QSM and 15\% of GTE (QG-3) \\
Coating with QSM and 20\% of GTE (QG-4) \\
\hline QSM, quince seed mucilage; GTE, green tea extract
\end{tabular}

\section{The pH values}

The $\mathrm{pH}$ values were assessed using digital $\mathrm{pH}$ meter (913 pH Meter, Metrohm, Switzerland).

\section{Color analysis}

Shrimp color was analyzed using colorimeter (Konica Minolta, CR400, Japan) and CIE Lab Scale $\left(\mathrm{L}^{*}, \mathrm{a}^{*}\right.$ and $\left.\mathrm{b}^{*}\right)$. To analyze total color differences between the three coordinates, the following formula was used (16):

$\Delta \mathrm{E}=\left[\Delta \mathrm{L}^{2}+\Delta \mathrm{a}^{*^{2}}+\Delta \mathrm{b}^{* 2}\right]^{1 / 2}$

\section{Total volatile basic nitrogen (TVB-N) analysis}

The TVB-N was assessed as described previously (Sun et al., 2014). Kjeldahl equipment with steam distillation of trichloroacetic acid was used to assess TVB-N. The TVB-N contents were represented as $\mathrm{mg}$ $\mathrm{N}(100 \mathrm{~g})^{-1}$ shrimp meat (7).

\section{Texture analysis}

Shrimps texture was analyzed for hardness and springiness using texture analyzer (TA. XT2i, Stable Micro System, Goldalming, UK) with a $12.7-\mathrm{mm}$ cylindrical probe at a speed of $0.5 \mathrm{~mm} \mathrm{~s}^{-1}$ (1).

\section{Microbiological analysis}

Method of Yuan et al. (2016) was used for the total aerobic plate count (TAC). After 1 min homogenization of the shrimps $(25 \mathrm{~g})$ at $8000 \mathrm{rpm}$ using sterile saline (225 ml, 85\%), the homogenizes were logarithmically diluted using sterile saline (85\%). To calculate the TAC, samples were incubated at $36{ }^{\circ} \mathrm{C}$ for $48 \mathrm{~h}$ after spread plating on nutrient agar. 
Microbial colonies were enumerated and presented as $\log \mathrm{CFU} \mathrm{g}^{-1}$ of fresh weight (8).

\section{Statistical analysis}

All experiments were carried out in triplicates and the average values were reported. Data analysis was carried out using SPSS Software v.19 (SPSS Inc., Chicago, IL, USA). One-way analysis of variance was used to investigate differences between the mean values of the samples. Tukey's multiple comparison test results were considered significant at $95 \%(p<$ $0.05)$.

\section{Results}

\section{The $\mathrm{pH}$ values}

Figure 1 shows changes in $\mathrm{pH}$ of the shrimps during storage at $4^{\circ} \mathrm{C}$. Initial $\mathrm{pH}$ of the shrimps was $6.97 \pm 0.2$, which was similar to other reports (4). As shown in Figure 1, pH of the shrimps in all samples increased during storage at $4{ }^{\circ} \mathrm{C}$. The increased rate of $\mathrm{pH}$ varied with various treatments. Increased $\mathrm{pH}$ values of the shrimps with the QSM and the QSM and GTE coatings were lower than those of control shrimps, similar to other reports (8). In this study, increased $\mathrm{pH}$ values in shrimps were significantly prevented by increased GTE concentrations. During storage, QS-G20 showed the lowest $\mathrm{pH}$, compared to control $(p<0.05)$. These results showed that the QSM and GTE coating could decrease spoilage and decomposition that caused quality loss of the shrimps.

\section{Total volatile basic nitrogen (TVB-N) analysis}

In general, TVB-N represents the quantity of ammonia and primary, secondary and tertiary amines. The TVB-N is the most important and common quality indicator of seafoods, which is widely used as an index of declines in muscle tissues caused by the activity of endogenous spoilage bacteria $(17,18)$. Figure 2 demonstrates changes in TVB-N of the shrimps treated by the QSM and the QSM and GTE coatings, compared to the control. Generally, the TVB-N of shrimps in all samples significantly increased during ten days of cold storage $(p<0.05)$. Thus, increased concentration of GTE decreased TVB-N. In the current study, the initial TVB-N in shrimps included $10.52 \mathrm{mg}(100 \mathrm{~g})^{-1}$. On Day 10 of cold storage, TVB-N included $57.54 \mathrm{mg}(100 \mathrm{~g})^{-1}$ and exceeded the spoilage value limit of shrimps. According to sanitary standards, TVB-N of fresh shrimps must be less than $300 \mathrm{mg} \mathrm{kg}^{-1}$ (4). The TVB$\mathrm{N}$ of QS-G20 included $42.91 \mathrm{mg}(100 \mathrm{~g})^{-1}$ at Day 10 of cold storage. Moreover, increases in TVB-N of the shrimps treated by the QSM coating were higher than increases in TVB-N of those treated by the QSM and GTE coating. These results have suggested that the QSM coating includes synergism in delaying TVB-N while used in combination with GTE.

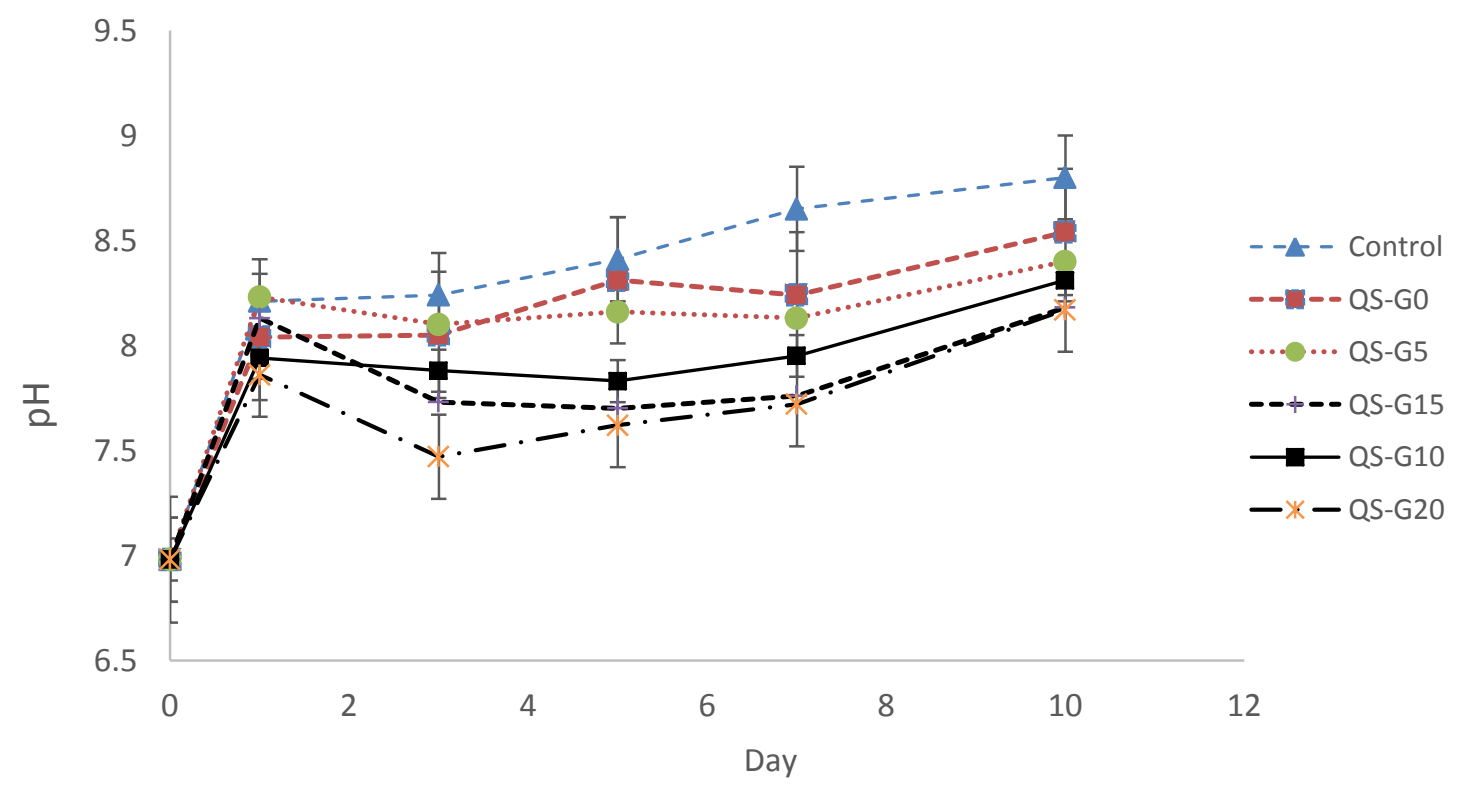

Figure 1. Combined effects of the QSM and GTE coating on $\mathrm{pH}$ of Pacific white shrimps during cold storage 


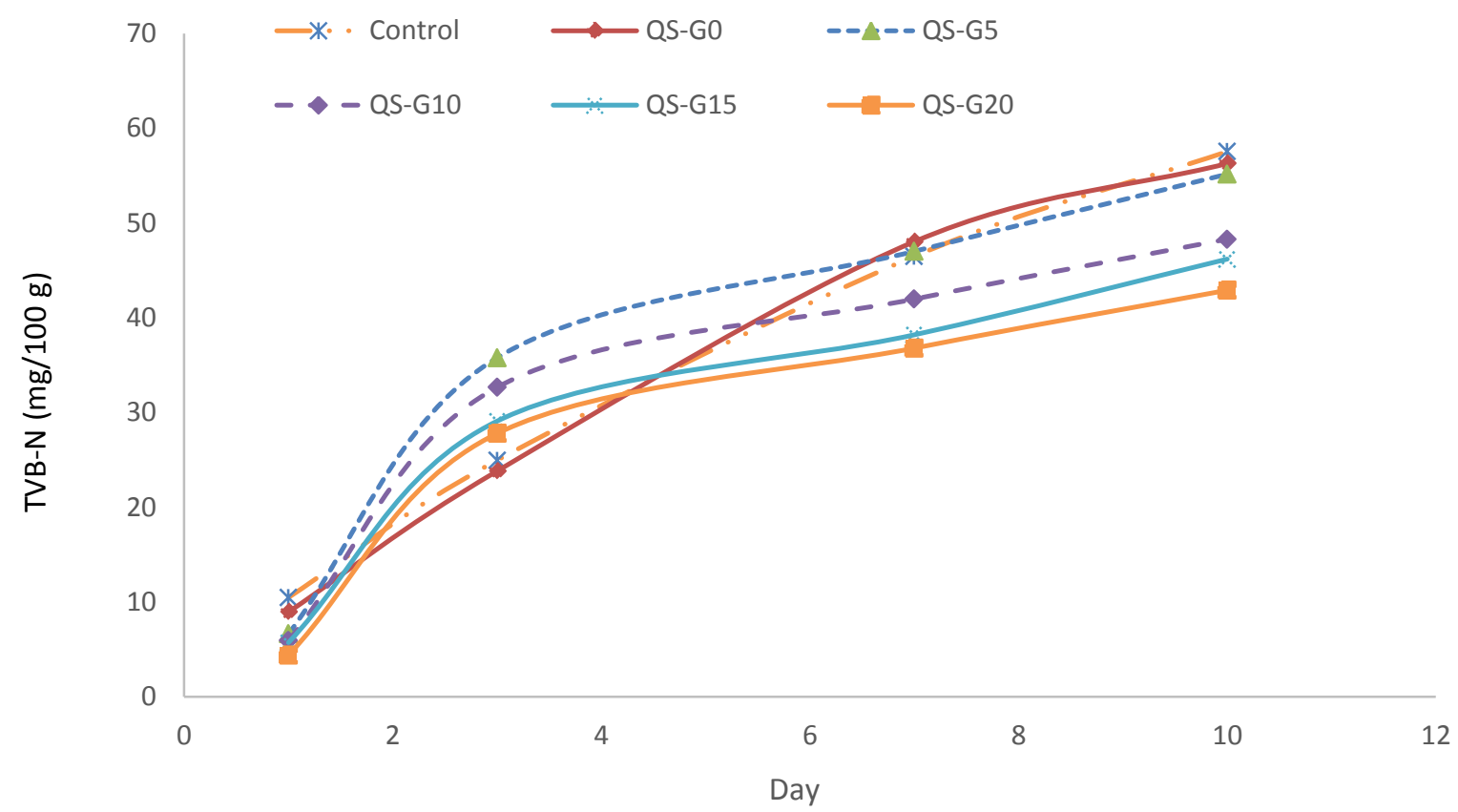

Figure 2. Combined effects of the QSM and GTE coating on TVB-N of Pacific white shrimps during cold storage

\subsection{Texture analysis}

The variation in texture features is one of the important quality attributes in seafood acceptability affected by several factors such as changes in $\mathrm{pH}$ values and deteriorations of proteins (19). Thus, the texture analysis is an important method to assess the effects of preservation methods on quality of shrimps. In general, hardness of the shrimps decreased in all treatments during cold storage, while increased concentration of GTE improved hardness of the shrimps, compared to the control (Figure 3a). Springiness of the shrimps in treatments is shown in Figure $3 \mathrm{~b}$. Springiness of the shrimps in all treatments decreased during cold storage. In contrast, springiness of the shrimps was improved by increasing concentration of the GTE during cold storage, compared to the control. The highest springiness value $(p<0.05)$ was seen in the treatment of QS-G20 on Day 10 of cold storage.

\subsection{Image analysis}

Effects of GTE concentration on color values of the shrimps during cold storage are shown in Table 2. Addition of GTE (from 0 to $20 \%$ ) to QSM coating solution significantly affected the $\mathrm{a}^{*}$ and $\mathrm{b}^{*}$ values of the shrimps $(p<0.05)$. The $\mathrm{a}^{*}$ value of the shrimps decreased from $-0.87 \pm 0.21$ to $-5.05 \pm 1.08$ (index of the attitude towards greenness) and the $b^{*}$ value increased from $1.44 \pm 0.84$ to $16.47 \pm 1.61$ (index to the attitude toward yellowness). Similar results have been reported by Siripatrawan and Harte (2010), who studied the incorporation of GTE into chitosan films (14). Higher $\Delta \mathrm{E}$ results showed more relative changes in shrimp color, compared to the initial shrimp color. After 10 days of cold storage, $\Delta \mathrm{E}$ included 1.9 for the control and 15.56 for the treatment of QS-G20 on Day 10 of cold storage. Moreover, $\Delta \mathrm{E}$ of the control sample was lowest than that of other samples at the end of cold storage. This possibly occurred due to the effects of coating on $a^{*}$ and $b^{*}$ values, which increased $\Delta \mathrm{E}$.

Table 2. Combined effects of the QSM and GTE coating on color parameters of Pacific white shrimps during cold storage. Means in the same column with different letters are significantly different $(p<0.05)$

\begin{tabular}{lccc}
\hline Treatment & $\mathrm{a}^{*}$ value & $\mathrm{b}^{*}$ value & $\Delta \mathrm{E}$ \\
\hline Control & $-0.87 \pm 0.1^{\mathrm{A}}$ & $1.44 \pm 0.83^{\mathrm{D}}$ & $0.09 \pm 0.04$ \\
QS-G0 & $-1.23 \pm 0.14^{\mathrm{A}}$ & $3.157 \pm 0.91^{\mathrm{CD}}$ & $5.44 \pm 0.28$ \\
QS-G5 & $-1.89 \pm 0.19^{\mathrm{B}}$ & $6.73 \pm 0.58^{\mathrm{BC}}$ & $8.64 \pm 1.1$ \\
GS-G10 & $-3.41 \pm 0.27^{\mathrm{C}}$ & $10.28 \pm 0.78^{\mathrm{B}}$ & $10.26 \pm 1.74$ \\
QS-G15 & $-3.46 \pm 0.18^{\mathrm{C}}$ & $10.43 \pm 0.94^{\mathrm{B}}$ & $10.33 \pm 1.29$ \\
QS-G20 & $-5.05 \pm 0.12^{\mathrm{D}}$ & $16.45 \pm 1.42^{\mathrm{A}}$ & $15.56 \pm 2.04$ \\
\hline
\end{tabular}




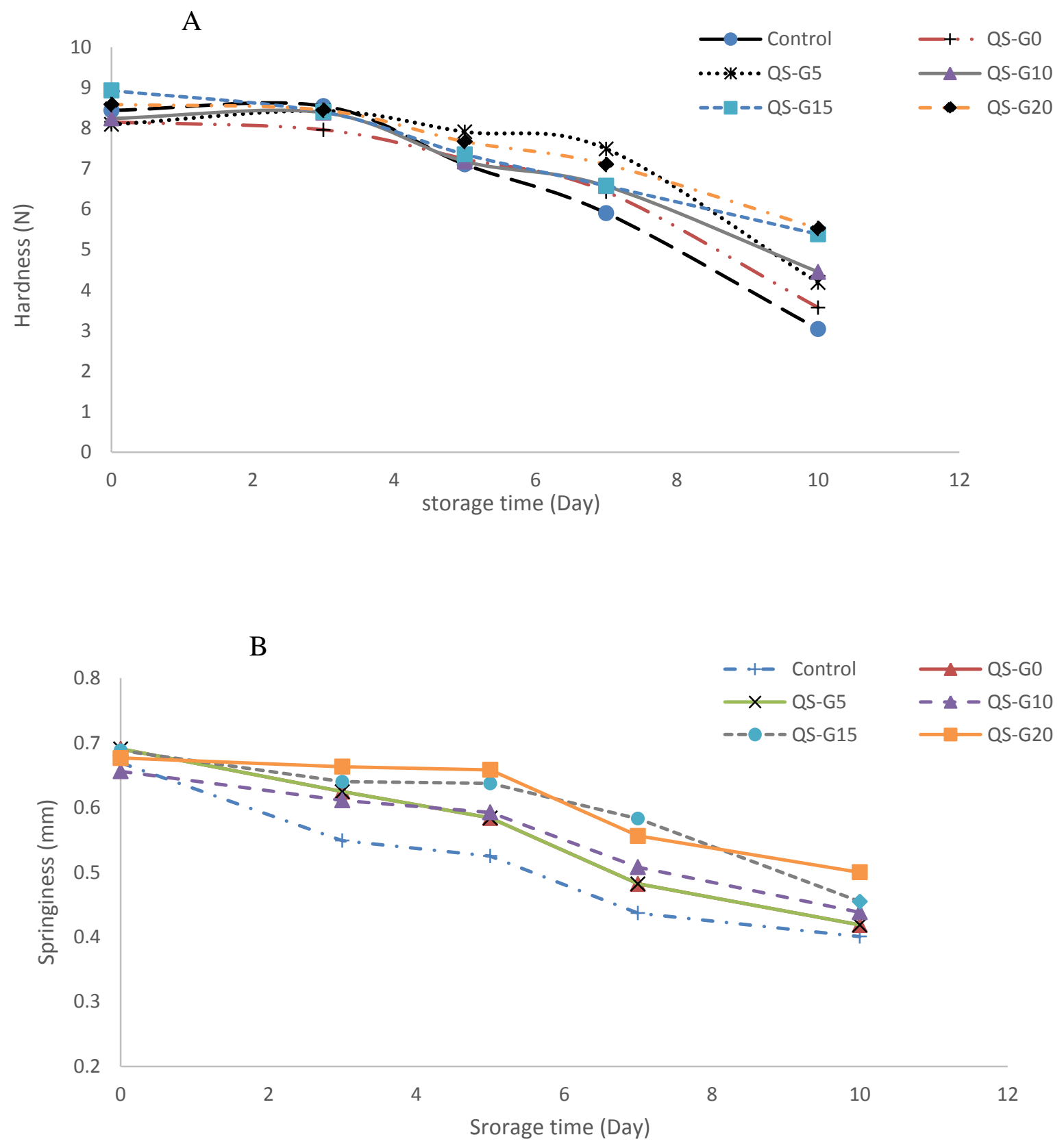

Figure 3. Combined effects of the QSM and GTE coating on texture variation of Pacific white shrimps during cold storage. A, hardness; $\mathrm{B}$, springiness

\subsection{Microbiological analysis}

The TAC of all treatments is shown in Figure 4. The TAC of all treatments increased throughout storage for ten days. Increases were mostly associated to control treatments while increased concentration of GTE decreased TAC. Generally, GTE is considered as a good source of polyphenolic compounds such as catechins, theaflavins, tannins and flavonoids with antioxidant and antimicrobial effects. Studies have shown the antimicrobial activity of GTE in agar diffusions (in vitro) and foods (in situ) (20-23). Initial TAC of the shrimps included $3.1 \log$ CFU g ${ }^{-1}$, which increased to $3.6 \log \mathrm{CFU} \mathrm{g}{ }^{-1}$ for control samples after 10 days of cold storage. The TAC of QS-G20 was significantly $(p<0.05)$ lower than that of control during storage. 


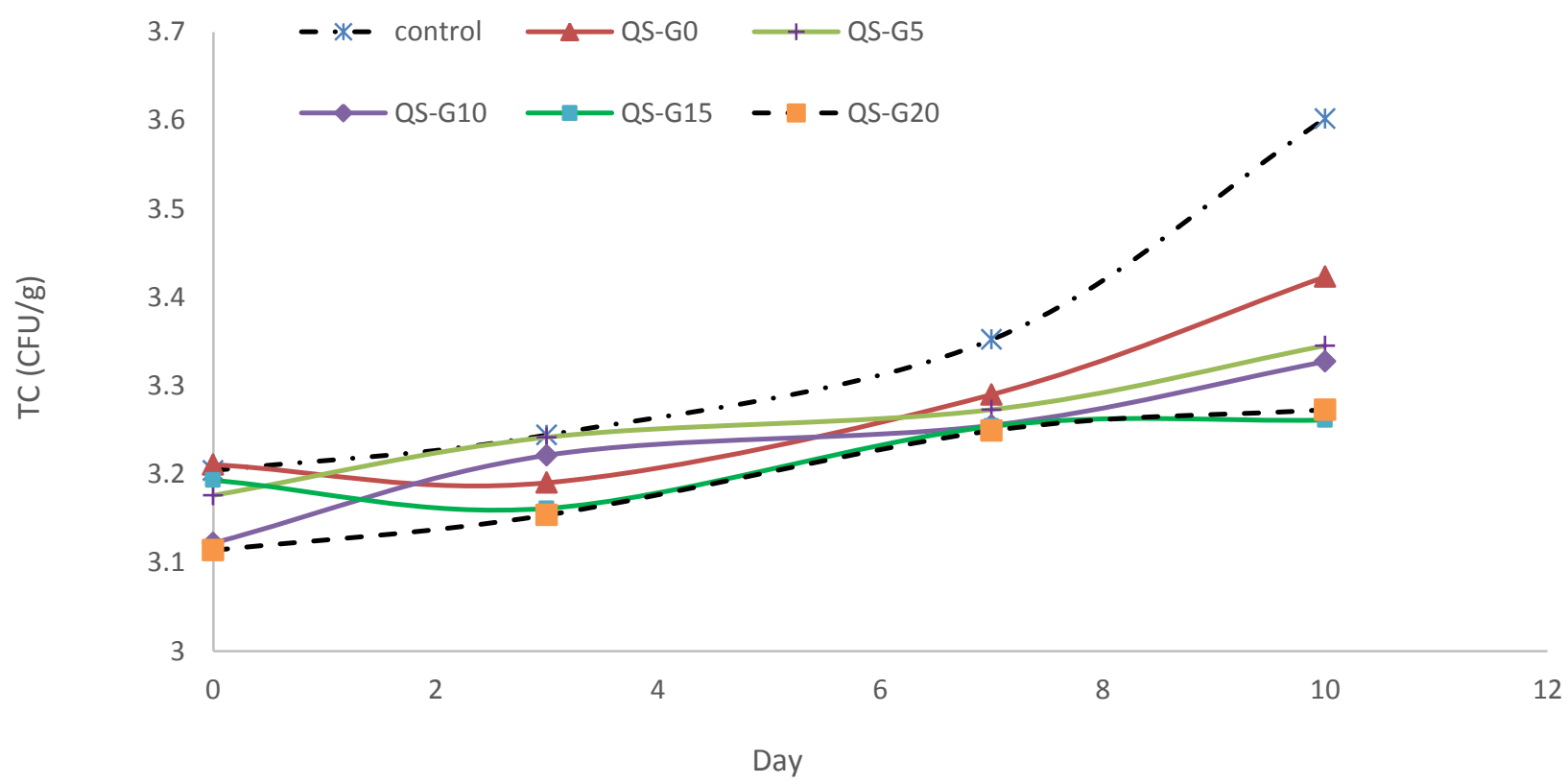

Figure 4. Total aerobic plate counts of the coated and control Pacific white shrimps during cold storage

\section{Discussion}

Mild primary decreased $\mathrm{pH}$ during storage was linked to migration from the coating to shrimps, which included an acid $\mathrm{pH}(5.9 \pm 0.1)$. The rate of increased $\mathrm{pH}$ varied within various treatments. Moreover, activity of bacteria or enzymatic actions could lead to the cumulation of basic compounds such as TVB-N, which changed $\mathrm{pH}$ in shrimps. In fact, TVB-N is directly linked to microbial spoilage in various species of shrimps during storage at cold conditions. The current results were similar to those from previous studies, reporting that TVB-N of the white shrimps treated with grape seed extracts decreased significantly (7). Generally, shrimp texture changes during storage due to the microbial activity. Microbial activity changes food organoleptic characteristics, which decreases the general acceptance of the foods in terms of color, odor and texture. Finding by the present study are similar to those by Li et al. (2013), who reported that sources of phenolic compounds such as grape seeds and teas could increase the shelf life and improve the textural characters of red drums (24). Furthermore, other findings by the current study are similar to findings by Basiri et al. (2015), who showed successful prevention of psychotropic bacterial growth in refrigerated shrimps treated with pomegranate peel extracts (25).

\section{Conclusion}

Results of this study have shown that QSM coating combined with GTE can improve the texture quality (e.g. total color differences $(\Delta \mathrm{E})$, hardness and springiness) of Pacific white shrimps during 10 days of cold storage. The QSM coating combined with GTE decreases TVC, $\mathrm{pH}$ and TVB-N of the Pacific white shrimps. In conclusion, results suggest that QSM coating with GTE can be used as an efficient natural method to preserve quality of shrimps during cold storage.

\section{Acknowledgement}

The authors would like to express their sincere thanks to Research Deputy of Agricultural Sciences and Natural Resources University of Khuzestan for the financial support (Grant No. 951/23).

\section{Financial disclosure}

No conflict of interest is declared. 


\section{References}

1. Jiang M, Liu S, Wang Y. Effects of antimicrobial coating from catfish skin gelatin on quality and shelf life of fresh white shrimp (Penaeus vannamei). Journal of food science. 2011;76(3):M204-M9.

2. Sharifimehr S, Soltanizadeh N, Goli SAH. Physicochemical properties of fried shrimp coated with bio-nano-coating containing eugenol and Aloe vera. LWT. 2019;109:33-9.

3. Kagawa M, Matsumoto M, Yoneda C, Mitsuhashi T, Hatae K. Changes in meat texture of three varieties of squid in the early stage of cold storage. Fisheries science. 2002;68(4):783-92.

4. Khazaei N, Esmaiili M, Emam-Djomeh Z. Application of active edible coatings made from basil seed gum and thymol for quality maintenance of shrimp during cold storage. Journal of the Science of Food and Agriculture. 2017;97(6):1837-45.

5. Nirmal NP, Benjakul S. Effect of ferulic acid on inhibition of polyphenoloxidase and quality changes of Pacific white shrimp (Litopenaeus vannamei) during iced storage. Food chemistry. 2009;116(1):323-31.

6. Nunak N, Schleining G. Instrumental textural changes in raw white shrimp during iced storage. Journal of Aquatic Food Product Technology. 2011;20(4):350-60.

7. Sun H, Lv H, Yuan G, Fang X. Effect of grape seed extracts on the melanosis and quality of Pacific white shrimp (Litopenaeus vannamei) during iced storage. Food Science and Technology Research. 2014;20(3):671-7.

8. Yuan G, Lv H, Tang W, Zhang X, Sun H. Effect of chitosan coating combined with pomegranate peel extract on the quality of Pacific white shrimp during iced storage. Food Control. 2016;59:818-23.

9. Salehi F. Characterization of new biodegradable edible films and coatings based on seeds gum: A review. Journal of Packaging Technology and Research. 2019:1-9.

10. Yu D, Regenstein JM, Xia W. Bio-based edible coatings for the preservation of fishery products: A review. Critical reviews in food science and nutrition. 2019;59(15):2481-93.

11.Jouki M, Yazdi FT, Mortazavi SA, Koocheki A. Quince seed mucilage films incorporated with oregano essential oil: physical, thermal, barrier, antioxidant and antibacterial properties. Food Hydrocolloids. 2014;36:9-19.

12. Noshad M, Nasehi B, Anvar A. Effect of active edible coating made by quince seed mucilage and green tea extract on quality of fried shrimps: physicochemical and sensory properties. Nutrition and Food Sciences Research. 2017;4(4):31-6.

13.Jouki M, Mortazavi SA, Yazdi FT, Koocheki A. Optimization of extraction, antioxidant activity and functional properties of quince seed mucilage by RSM. International journal of biological macromolecules. 2014;66:113-24.

14. Siripatrawan U, Harte BR. Physical properties and antioxidant activity of an active film from chitosan incorporated with green tea extract. Food Hydrocolloids. 2010;24(8):770-5.

15.Sabaghi M, Maghsoudlou Y, Khomeiri M, Ziaiifar AM. Active edible coating from chitosan incorporating green tea extract as an antioxidant and antifungal on fresh walnut kernel. Postharvest Biology and Technology. 2015;110:224-8.

16. Islam M, Saha T, Monalisa K, Hoque M. Effect of starch edible coating on drying characteristics and antioxidant properties of papaya. Journal of Food Measurement and Characterization. 2019;13(4):2951-60.

17. Nirmal NP, Benjakul S. Effect of catechin and ferulic acid on melanosis and quality of Pacific white shrimp subjected to prior freeze-thawing during refrigerated storage. Food Control. 2010;21(9):126371.

18. Gharibzahedi SMT, Mohammadnabi S. Effect of novel bioactive edible coatings based on jujube gum and nettle oil-loaded nanoemulsions on the shelf-life of Beluga sturgeon fillets. International journal of biological macromolecules. 2017;95:769-77.

19.Bouaziz F, Koubaa M, Neifar M, Zouari-Ellouzi S, Besbes S, Chaari F, et al. Feasibility of using almond gum as coating agent to improve the quality of fried potato chips: Evaluation of sensorial properties. LWT-Food Science and Technology. 2016;65:800-7.

20. de Lacey AL, López-Caballero M, Montero P. Agar films containing green tea extract and probiotic bacteria for extending fish shelf-life. LWT-Food Science and Technology. 2014;55(2):559-64.

21. McCarthy S, Caporali A, Enkemann S, Scaltriti M, Eschrich S, Davalli P, et al. Green tea catechins suppress the DNA synthesis marker MCM7 in the TRAMP model of prostate cancer. Molecular oncology. 2007;1(2):196-204.

22. Mitsumoto M, O’Grady MN, Kerry JP, Buckley DJ. Addition of tea catechins and vitamin $\mathrm{C}$ on sensory evaluation, colour and lipid stability during chilled storage in cooked or raw beef and chicken patties. Meat Science. 2005;69(4):773-9. 
23.Perumalla A, Hettiarachchy NS. Green tea and grape seed extracts-Potential applications in food safety and quality. Food Research International. 2011;44(4):827-39.

24.Li T, Li J, Hu W, Li X. Quality enhancement in refrigerated red drum (Sciaenops ocellatus) fillets using chitosan coatings containing natural preservatives. Food Chemistry. 2013;138(2):821-6.
25. Basiri S, Shekarforoush SS, Aminlari M, Akbari S. The effect of pomegranate peel extract (PPE) on the polyphenol oxidase (PPO) and quality of Pacific white shrimp (Litopenaeus vannamei) during refrigerated storage. LWT-Food Science and Technology. 2015;60(2):1025-33. 